\title{
Características químicas y microbiológicas del ensilado de cáscara de cacao (Theobroma cacao L) tratada con yogur natural
}

\author{
Chemical and microbiological characteristics of cocoa shell silage \\ (Theobroma cacao $\mathrm{L}$ ) treated with natural yogurt
}

\author{
Willan Caicedo ${ }^{1,2,3}$, Luis Caicedo ${ }^{1}$
}

\section{Resumen}

Se determinaron las características químicas y microbiológicas del ensilado de cáscara de cacao (Theobroma cacao L) tratado con yogur natural como fuente de inóculo para uso en animales de interés zootécnico. Se emplearon 25 microsilos plásticos de $1 \mathrm{~kg}$ de capacidad, y se evaluaron en los días 1, 4, 8, 15 y 30 de fermentación. Los indicadores químicos fueron el $\mathrm{pH}$, materia seca (MS), proteína bruta $(\mathrm{PB})$, fibra bruta $(\mathrm{FB})$, cenizas, extracto etéreo (EE), extractos libres de nitrógeno (ELN) y energía bruta (EB). Los indicadores microbiológicos fueron la presencia o ausencia de Escherichia coli, Clostridium spp y Salmonella spp. El ensilado presentó un $\mathrm{pH}$ idóneo y un buen contenido de MS, PB, cenizas, ELN, EB y bajo nivel de EE. Los análisis microbiológicos fueron negativos para los microorganismos en evaluación. La combinación de cáscara de cacao molida, sal mineral, melaza, carbonato de calcio y yogur natural produjo un ensilado de apreciada calidad nutricional para uso en la alimentación animal.

Palabras clave: componentes químicos, cáscara de cacao, ensilaje, recurso alternativo

\footnotetext{
${ }^{1}$ Facultad de Ciencias de la Tierra, Universidad Estatal Amazónica, Puyo, Pastaza, Ecuador

${ }^{2}$ Granja Agropecuaria Caicedo, Puyo, Pastaza, Ecuador

${ }^{3}$ E-mail: orlando.caicedo@yahoo.es
}

Recibido: 29 de marzo de 2021

Aceptado para publicación: 5 de noviembre de 2021

Publicado: 22 de diciembre de 2021

CLos autores. Este artículo es publicado por la Rev Inv Vet Perú de la Facultad de Medicina Veterinaria, Universidad Nacional Mayor de San Marcos. Este es un artículo de acceso abierto, distribuido bajo los términos de la licencia Creative Commons Atribución 4.0 Internacional (CC BY 4.0) [https:// creativecommons.org/licenses/by/4.0/deed.es] que permite el uso, distribución y reproducción en cualquier medio, siempre que la obra original sea debidamente citada de su fuente original 
The chemical and microbiological characteristics of cocoa shell silage (Theobroma cacao L) treated with natural yogurt as an inoculum source for use in animals of zootechnical interest were determined. A total of 25 plastic bags of $1 \mathrm{~kg}$ capacity were used, and they were evaluated on days 1, 4, 8, 15 and 30 of fermentation. The chemical indicators were $\mathrm{pH}$, dry matter (DM), crude protein (CP), crude fibre (CF), ash, ethereal extract (EE), nitrogen-free extracts (NFE) and crude energy (CE); and the microbiological indicators were the presence or absence of Escherichia coli, Clostridium spp and Salmonella spp. The silage presented an ideal $\mathrm{pH}$ and a good content of DM, CP, ash, NFE, CE and a low level of EE. The microbiological analyses were negative for the microorganisms under evaluation. The combination of ground cocoa shell, mineral salt, molasses, calcium carbonate and natural yogurt produced a silage of appreciated nutritional quality for use in animal feed.

Key words: alternative resource, chemical components, cocoa shell, silage

\section{INTRODUCCIÓN}

En el cultivo de frutas se produce una gran cantidad de residuos que provocan serios problemas de contaminación ambiental (Sánchez-Santillán et al., 2019). Ecuador está catalogado como uno de los principales productores de cacao (Theobroma cacao L) a nivel mundial. Se extraen las semillas de la fruta para el procesamiento de chocolate y derivados, quedando la cáscara como un residuo poco valorado como alimento animal, de allí que es usualmente desechada en el campo de cultivo, iniciando un proceso de descomposición de la materia orgánica que contribuye a la presentación de plagas y enfermedades (Sánchez et al., 2015).

Makinde et al. (2019) manifiestan que el alto costo de los piensos y la gestión de la alimentación siguen siendo desafíos sin resolver que enfrenta la producción ganadera a nivel mundial, concretamente en los países en desarrollo. De hecho, más de la mitad del costo de producción está asociados a la alimentación, de allí la importancia para la ciencia ganadera de explorar recursos no convencionales para el uso eficiente de los ali- mentos para reducir costos y aumentar la productividad. Una de estas estrategias constituye el uso de alimentos alternativos o no convencionales como los subproductos del cacao (Hidayat et al., 2018). La cáscara de cacao posee un buen contenido nutricional, así como también, es una fuente rica en componentes bioactivos (antioxidantes) que pueden ejercer efectos benéficos en la salud de humanos y animales (Balentiæ et al., 2018; Pavlovi et al., 2020; Rojo-Poveda et al., 2020).

Para el aprovechamiento de los subproductos agrícolas y agroindustriales existen técnicas de conservación que permiten mejorar el valor nutricional de los alimentos; entre estas, el ensilado (Caicedo et al., 2019, 2020). Mediante el ensilado se puede obtener un alimento estable con una alta recuperación de materia seca, energía y nutrientes altamente digestibles en comparación con la materia prima natural. En este proceso biotecnológico se emplean inóculos de bacterias y levaduras para acidificar el medio y reducir el $\mathrm{pH}$ prontamente para garantizar una buena conservación de la materia orgánica por un largo periodo de tiempo (Nisa et al., 2019). No obstante, existe escaso aprovecha- 
miento de estos alimentos por el poco conocimiento técnico de las personas que manejan estos residuos, desestimándose una fuente de alimento alternativa para los animales (Borrás-Sandoval et al., 2017). Por lo tanto, el objetivo de este estudio fue determinar las características químicas y microbiológicas del ensilado de cáscara de cacao (Theobroma cacao L) tratado con yogur natural como fuente de inóculo para uso en animales de interés zootécnico.

\section{Materiales y Métodos}

La investigación se ejecutó en la Región Amazónica Ecuatoriana (RAE). La zona se encuentra a una altitud de $900 \mathrm{~m}$ y tiene un clima subtropical húmedo, con temperaturas que varían entre 20 y $28{ }^{\circ} \mathrm{C}$, precipitaciones pluviales de $4000-4500 \mathrm{~mm} / \mathrm{año} \mathrm{y} 87 \%$ de humedad relativa (INAMHI, 2014).

Se recogió cáscara de cacao en el Centro de Investigación Posgrado y Conservación Amazónica (CIPCA) de la Universidad Estatal Amazónica (UEA), ubicado entre los cantones Santa Clara y Carlos Julio Arose-mena Tola. El material colectado se llevó a las instalaciones de la «Granja Agrope-cuaria Caicedo» ubicadas en la parroquia Tarqui, cantón Pastaza, Ecuador. La cáscara se lavó con abundante agua potable, dejándose escurrir por 10 minutos bajo sombra, para luego ser molida en estado fresco con un molino de martillo, provisto de cuchillas y criba de $2 \mathrm{~cm}$.

La cáscara molida se llevó al laboratorio de Microbiología de la UEA, donde se realizó una mezcla con los demás ingredientes: cáscara de cacao molida (96\%), sal mineral $(0.5 \%)$, melaza (2\%), carbonato de calcio $(0.5 \%)$, yogur natural $(1 \%)$. La mezcla se puso en 25 microsilos plásticos, con capacidad para $1 \mathrm{~kg}$. La medición de los indicadores químicos y microbiológicos se hizo en los días 1, 4, 8, 15 y 30. La preparación de los microsilos se hizo en una sola oportunidad.
La medición del $\mathrm{pH}$ se hizo en cinco microsilos por cada día de estudio. empleando extracto acuoso compuesto por una fracción de $25 \mathrm{~g}$ de ensilado y $250 \mathrm{ml}$ de agua destilada, de acuerdo con la metodología de Cherney y Cherney (2003).

Para la determinación de los componentes químicos del ensilado se recolectaron dos muestras al azar de $1 \mathrm{~kg}$ de los microsilos del día 8 de fermentación. Se comprobó: materia seca (MS), fibra bruta (FB), cenizas, proteína bruta (PB), extracto etéreo (EE) y extractos libres de nitrógeno (ELN) de acuerdo con la AOAC (2005) y la energía bruta (EB) por calorimetría.

Para la comprobación microbiológica del ensilado se recolectaron dos muestras al azar de $500 \mathrm{~g}$, en cada tiempo de fermentación, y se comprobó la probable presencia de Escherichia coli, Clostridium spp y Salmonella spp siguiendo los procedimientos de la AOAC (2016).

En los datos de componentes químicos se calculó la media y la desviación estándar. Para los datos de $\mathrm{pH}$ e indicadores microbiológicos, el experimento se manejó de acuerdo con un diseño completamente al azar. Los datos se analizaron mediante un análisis de varianza y la diferencias entre medias mediante la prueba de Duncan $(\mathrm{p}<0,05)$, utilizando el programa estadístico Infostat (Di Rienzo et al., 2017).

\section{Resultados}

La variación del pH del ensilado de cáscara de cacao durante el periodo de evaluación se muestra en el Cuadro 1. El mayor valor $(\mathrm{p}<0.05)$ se presentó el día 1 , siendo estable entre los días 4 y 30 (4.47-4.47).

En el Cuadro 2 se muestran los componentes nutricionales del ensilado de cáscara de cacao. El ensilado presentó un apreciable 
Cuadro 1. Comportamiento del $\mathrm{pH}$ en el ensilado de cáscara de cacao (Theobroma cacao L)

\begin{tabular}{cc}
\hline Días de ensilado & $\mathrm{pH}$ \\
\hline 1 & $5.61^{\mathrm{a}}$ \\
4 & $4.47^{\mathrm{b}}$ \\
8 & $4.46^{\mathrm{b}}$ \\
15 & $4.47^{\mathrm{b}}$ \\
30 & $4.47^{\mathrm{b}}$ \\
\hline
\end{tabular}

$a, b$ Letras distintas muestran diferencias a nivel de $p<0.05$

Error estándar $=0.01$

contenido de MS, PB, ELN, EE, EB y bajo tenor de EE. No se encontró Escherichia coli, Clostridium spp o Salmonella spp durante los días de evaluación.

\section{Discusión}

El valor más alto de $\mathrm{pH}$ del día 1 se debe a la escasa producción de ácido láctico dentro de los microsilos (García et al., 2020). Sin embargo, a partir de las $96 \mathrm{~h}$ (día 4) el pH se estabilizó debido a la acidificación del medio por la inclusión de carbohidratos de fácil asimilación (melaza) (Nkosi y Meeske, 2010; Castaño y Villa, 2017) y a la fuente de inóculo inicial (Gunawan et al., 2015; Muck et al., 2018). En el proceso de ensilado, el ácido láctico (pKa de 3.86), producido por las bacterias ácido-lácticas, suele ser el ácido que se encuentra en la concentración más alta en los silos, y es el que más contribuye a la disminución del $\mathrm{pH}$ durante la fermentación. Este ácido es aproximadamente 10-12 veces más fuerte que cualquiera de los otros ácidos principales que se encuentran en los ensilajes [por ejemplo, el ácido acético (pKa de 4.75) y el ácido propiónico (pKa de 4.87)] (Kung et al., 2018). En el medio ácido se desarrollan rápidamente los microrganismos conservadores de la materia prima (Guan et al., 2020), limi- tando el crecimiento de microrganismos putrefactivos intolerantes a un $\mathrm{pH}$ bajo del silo que podrían afectar negativamente el rendimiento de los animales (Kung et al., 2018).

Los valores de la composición química (Cuadro 2) fueron similares a los reportados por Chafla et al. (2016) en cáscara de cacao de tres cantones de la provincia de Pastaza, Ecuador. Los procesos de fermentación idóneos tienen la ventaja de conservar los nutrientes en los residuos agrícolas por tiempo prolongado para uso en la alimentación de animales (Caicedo et al., 2016). Por otro lado, la cáscara en estado natural posee un alto contenido de teobromina que puede afectar el desempeño productivo de algunas especies de animales (Silva et al., 2005). En este sentido, Caicedo et al. (2017) y Ortiz et al. (2019) reportan que a través de la fermentación se puede limitar y/o disminuir el contenido de metabolitos secundarios de la materia prima.

Omotoso et al. (2018) investigaron los efectos del ensilaje de la harina de cáscara de cacao tratada con urea y combinada con harina de cáscara de yuca podría mejorar el rendimiento de crecimiento de las cabras sin efectos adversos. Asimismo, Fridarti et al.

Cuadro 2. Composición química del ensilado de cáscara de cacao (Theobroma cacao L) en base seca

\begin{tabular}{lc}
\hline Nutrientes & Media \pm D.E \\
\hline Materia seca, MS (\%) & $18.61 \pm 0.1$ \\
Proteína bruta, PB (\%) & $7.89 \pm 0.01$ \\
Fibra bruta, FB, (\%) & $22.79 \pm 0.45$ \\
Extracto etéreo, EE (\%) & $2.76 \pm 0.01$ \\
$\begin{array}{l}\text { Cenizas (\%) } \\
\text { Extracto libre de } \\
\text { nitrógeno ELN (\%) }\end{array}$ & $5.45 \pm 0.31$ \\
$\begin{array}{l}\text { Energía bruta, EB } \\
\text { (kcal/kg MS) }\end{array}$ & $3391.5 \pm 1.36 \pm 0.05$ \\
\hline
\end{tabular}


(2017) indican que las hojas y la cáscara del fruto fermentado de cacao pueden reemplazar el $50 \%$ del forraje y aumentar el rendimiento productivo de las ovejas. En otro estudio, Ogunsipe et al. (2017) en su estudio con lechones destetados alimentados con niveles crecientes (desde 0 hasta $40 \%$ ) de harina de cáscara de cacao en sustitución del maíz no se afectó la ingesta de alimento, pero la ganancia de peso disminuyó cuando la harina de cáscara de cacao excedió el $20 \%$ de la dieta.

La fermentación microbiana en el silo produce una variedad de productos finales y puede influir sobre muchos aspectos nutritivos y sensoriales del silo. La ausencia de $E$. coli, Clostridium spp y Salmonella spp en el ensilado de cáscara de cacao es favorable para garantizar la inocuidad del alimento y no comprometer la salud de los animales (Johansson et al., 2005). Estos resultados son similares a los reportados por Caicedo et al. (2016) en ensilados de recursos agrícolas (tubérculos de taro), quienes informaron la ausencia de coliformes totales, E. coli, Clostridium spp y Salmonella spp durante los días de investigación.

\section{Conclusión}

La combinación de cáscara de cacao molida, sal mineral, melaza, carbonato de calcio y yogur natural produjo un ensilado de apreciada calidad nutricional para uso en la alimentación animal.

\section{Literatura Citada}

1. [AOAC] Association of Official Agricultural Chemists. 2005. Official Methods of Analysis. $18^{\text {th }}$ ed. USA: $\mathrm{AOAC}$

2. [AOAC] Association of Official Agricultural Chemists. 2016. Official methods of analysis of AOAC Inter- national. $20^{\text {th }}$ ed. USA: AOAC. [Internet]. Disponible en: http://www.directtextbook.com/isbn/9780935584875

3. Balentice JP, Aèkar D, Joki S, Jozinoviae A, Babice J, Milièevia B, Šubarice D, Pavlovice N. 2020. Cocoa shell: a by-product with great potential for wide application. Molecules 23: 1404. doi: 10.3390/molecules23061404

4. Borrás-Sandoval L, Valiño E, Elias A. 2017. Evaluación del efecto de la inclusión de materiales fibrosos en la fermentación en estado sólido de residuos poscosecha de papa (Solanum tuberosum) inoculado con preparado microbial. REDVET 18(8). [Internet]. Disponible en: https://www.redalyc.org/pdf/636/ 63652581011.pdf

5. Caicedo W, Rodríguez, $R$, Lezcano $P$, Ly J, Valle S, Flores L, Ferreira FNA. 2016. Physicochemical and biological indicators in silages of taro (Colocasia esculenta (L) Schott) tubers for animal feeding. Cuban J Agr Sci 50: 121-129.

6. Caicedo W, Rodríguez $R$, Lezcano P, Ly J, Vargas JC, Uvidia $H$, Valle $S$, Flores L. 2017. Characterization of antinutrients in four silages of taro (Colocasia esculenta (L) Schott) for pigs. Technical note. Cuban J Agr Sci 51: 79-83.

7. Caicedo W, Ferreira $F N$, Viáfara $D$, Guamán A, Sócola C, Pérez M, Díaz L, Ferreira WM. 2019. Evaluación química y digestibilidad fecal de cerdos en crecimiento alimentados con banano orito (Musa acuminata AA) fermentado en estado sólido. Livestock Res Rural Dev 31(11). [Internet]. Disponible en: http:// www.lrrd.org/lrrd31/11/orlan31170.html

8. Caicedo W, Viáfara D, Pérez, $M$, Ferreira F N A, Pico K, Cachago K, Valle S, Ferreira W M. 2020. Technical note on physiochemical and organoleptic indicators of orito banana (Musa acuminata $\mathrm{AA}$ ) rachis silage. Cuban $\mathrm{J}$ Agr Sci 54: 237-241. 
9. Castaño GA, Villa LM. 2017. Use of whey and molasses as additive for producing silage of Cuba OM-22 (Cenchrus purpureus x Cenchrus glaucum). Cuban J Agr Sci 51: 61-70.

10. Chafla AL, Rodríguez Z, Boucourt R, Torres V. 2016. Bromatological characterization of cocoa shell (Theobroma cacao), from seven cantons of the Amazonia, Ecuador. Cuban J Agr Sci 50:245-252.

11. Cherney JH, Cherney DJR. 2003. Assessing silage quality. In: Buxton DR, Muck RE, Harrison JH (eds). Silage science and technology, Wisconsin, USA: American Society of Agronomy. p 141-198.

12. Di Rienzo JA, Casanoves F, Balzarini MG, González L, Robledo CW. 2017. InfoStat. [Internet]. Disponible en: http:/ /www.infostat.com.ar/

13. Fridarti NJ, Zain M, Ningrat RWS. 2017. The use of waste cacao (Theobroma cacao L) fermentation as a substitute for forage to cattle sheep performance. Scholars J Agric Vet Sci 4: 230-235. doi: 10.31227/osf.io/g69nz

14. García HY, Sosa Cossio D, González González L, Dustet Mendoza JC. 2020. Caracterización química, física y microbiológica de alimentos fermentados para su uso en la producción animal. Livestock Res Rural Dev 32(7). [Internet]. Disponible en: http:// www.lrrd.org/lrrd32/7/Yaneis32105.html

15. Guan H, Shuai Y, Yan Y, Ran $Q$, Wang X, Li D, Cai Y, Zhang X. 2020. Microbial community and fermentation dynamics of corn silage prepared with heat-resistant lactic acid bacteria in a hot environment. Microorganisms 8: 719. doi: 10.3390/microorganisms8050719

16. Gunawan S, Widjaja T, Zullaikah S, Ernawati L, Istianah N, Aparamarta HW, Prasetyoko D. 2015. Effect of fermenting cassava with Lactobacillus plantarum, Saccharomyces cereviseae, and Rhizopus oryzae on the chemical composition of their flour. Int Food Res J 22: 1280-1287.
17. Hidayat M, Pratama HY, Martono E. 2018. Utilization of cacao pod husk silage as cattle mixture at Taluditi, Pohuwatu Regency, Gorontalo Province. IOP C Ser Earth Env 119: 012011. doi: 10.1088/1755-1315/119/1/012011

18. [INAMHI] Instituto Nacional de Meteorología e Hidrología. 2014. Anuario meteorológico. Quito, Ecuador. 28 p. [Internet]. Disponible en: http://www.serviciometeorologico.gob.ec/wp-content/uploads/anuarios/meteorologicos/ Am\%202011.pdf

19. Johansson M, Emmoth E, Salomonsson AC, Albihn A. 2005. Potential risks when spreading anaerobic digestion residues on grass silage crops - survival of bacteria,_moulds and viruses. Grass Forage Sci 60: 175-185. doi: 10.1111/ j.1365-2494.2005.00466.x

20. Kung L, Shaver RD, Grant RJ, Schmidt RJ. 2018. Silage review: Interpretation of chemical, microbial, and organoleptic components of silages. J Dairy Sci 101: 4020-4033. doi: 10.3168/ jds.2017-13909

21. Makinde OJ, Okunade SA, Opoola E, Sikiru AB, Ajide SO, Elaigwu S. 2019. Exploration of cocoa (Theobroma ca$\mathrm{cao}$ ) by-products as valuable potential resources in livestock feeds and feeding systems. In: Theobroma cacao Deploying science for sustainability of global cocoa economy. IntechOpen. doi: 10.5772/intechopen.87871.

22. Muck RE, Nadeau EMG, McAllister TA, Contreras-Govea FE, Santos MC, Kung LJ. 2018. Silage review: recent advances and future uses of silage additives. J Dairy Sci 101: 3980-4000. doi: $10.3168 /$ jds.2017-13839

23. Nisa K, Rosyida VT, Nurhayati S, Indrianingsih $A W$, Darsih $C$, Apriyana W. 2019. Total phenolic contents and antioxidant activity of rice bran fermented with lactic acid bacteria. IOP C Ser Earth Env 251: 012020. doi: $10.1088 / 1755-1315 / 251 / 1 / 012020$ 
24. Nkosi BD, Meeske R. 2010. Effects of whey and molasses as silage additives on potato hash silage quality and growth performance of lambs. S Afr J Anim Sci 40: 229-237. doi: 10.4314/sajas.v40i3.7

25. Ogunsipe MH, Ibidapo I, Oloruntola OD, Agbede JO. 2017. Growth performance of pigs on dietary cocoa bean shell meal. Livestock Res Rural Dev 29(1). [Internet]. Disponible en: http://www.lrrd.org//rrd29/1/ogun29018.html

26. Omotoso OB, Bello IA, Fajemisin AN. 2018. Effects of differently treated Theobroma cacao pod husk silage and cassava peel meals on nutrient utilization and weight gain of West African dwarf goats. Anim Res Int 15: 3020-3033.

27. Ortiz J, Chungara M, Ibieta G, Alej I, Tejeda L, Peralta C, Aliaga-Rossel E, et al. 2019. Determinación de teobromina, catequina, capacidad antioxidante total y contenido fenólico total en muestras representativas de cacao amazónico boliviano y su comparación antes y después del proceso de fermentación. Rev Boliviana Quím 36: 40-50.

28. Pavlovi N, Joki S, Jakovljevi M, Blaži M, Molnar M. 2020. Green extraction methods for active compoundsfrom food waste - cocoa bean shell. Foods 9: 140. doi: $10.3390 /$ foods 9020140

29. Rojo-Poveda O, Barbosa-Pereira L, Zeppa G, Stévigny C. 2020. Cocoa bean shell - a by-product with nutritional properties and biofunctional potential. Nutrients 12: 1123. doi: 10.3390/ nu12041123

30. Sánchez M, Jaramillo E, Ramírez I. 2015. Enfermedades del cacao. Ecuador: Univ. Técnica de Machala. $153 \mathrm{p}$.

31. Sánchez-Santillán P, Herrera-Pérez J, Torres-Salado N, Almaraz-Buendía I, Reyes-Vázquez I, Rojas-García AR, Gómez-Trinidad M, et al. 2019. Chemical composition and in vitro fermentation of ripe mango silage with molasses. Agroforest Syst 94: 1511-1519.

32. Silva HG, Pires AJV, Silva FF, Veloso CM, Cravalho GGP, Cezário AS, Santos CC. 2005. Farelo de cacau (Theobroma cacao L) e torta de dendê (Elaeis guineensis, Jacq) na alimentação de cabras em lactação: consumo e produção de leite. Rev Bras Zootecn 34: 17861794. doi: 10.1590/S1516-35982005000500040 